\title{
CTOS BOARD OF DIRECTORS 2000-2001
}

Jaap Verweij, MD President

Lee Helman, MD Vice President

Mark Gebhardt, MD Secretary

Robert S. Benjamin, MD Treasurer

Brian O'Sullivan, MD Past President

J. Sybil Biermann, MD (1998-2001)

Vivien Bramwell-Wesley, MD (1998-2001)

Ian Judson, MD (1998-2001)

Claude Turc-Carel, MD (1998-2001)

Frits Van Coevorden, MD (1998-2001)

Franco Gherlinzoni, MD (1999-2002)

Michael Simon, MD (1999-2002)

Scott Nelson, MD (1999-2002)

Martine van Glabbeke, MD (1999-2002)

Ephraim Casper, MD (2000-2003)

Andrew Huvos, MD (2000-2003)

Martin Robinson, MD (2000-2003)

Junya Toguchida, MD (2000-2003) 


\section{CTOS Meeting Program}

\section{Thursday, November 1, 2001}

\section{1:00-5:00 p.m. Registration, Set Up Posters}

6:00-7:00 p.m. Welcome Reception

\section{Friday, November 2, 2001}

7:00 a.m. Registration, Breakfast for meeting attendees

7:00 a.m. Executive Committee Meeting

8:00 a.m. Welcome, Opening Announcements: Jaap Verweij, CTOS President

8:15 a.m. PEDIATRIC SARCOMAS: Chairmen: Lee Helman (USA) \& Herbert Jurgens (Germany)

8:15 a.m.
8:30 a.m.
8:45 a.m.
9:00 a.m.
9:15 a.m.

9:30 a.m.
Herbert Jurgens: Pediatric sarcoma: state of the art

Tim Triche: From gene activation to signal transduction treatment Lenny Wexler: Irinotecan in refractory RMS

Peter Anderson: Inhalation GMCSF for treatment of pulmonary metastases

Abstract number $40 \mathrm{Her}-2 / \mathrm{NEU}$ staining in osteosarcoma: association with increased risk of metastatis-Holly Zhou, Rob Goldsby, R. Lor Randall, Lynn Smith, Cheryl M. Coffin Departments of Pathology, Pediatrics, Orthopedics, and Radiation Oncology, University of Utah Summary and Panel Discussion: Where do we go from here? Lee Helman

10.00 a.m. Coffee Break, Poster Viewing

10:15 a.m. BONE TUMORS: Chairmen: Peter Choong (Australia) \& Thor Alvegard (Sweden)

10:15 a.m.

10:30 a.m.

10:45 a.m.

11:00 a.m.

11:15 a.m.

11:30 a.m.

11:45 a.m.

12:00 p.m
Linda Granowetter: US Ewing's sarcoma randomized study of higher dose therapy

Kristy Weber: Implications and management of local relapse in Ewing's sarcoma

Peter Choong: The role of functional nuclear scanning in sarcoma management

Sigbjörn Smeland: An overview of treatments results in osteosarcoma: is there a need for a global collaboration?

Abstract number 87 Reconstruction of the pelvis following resection of tumors about the acetabulum-R. L. Satcher, Jr., R. J. O’Donnell, J. O. Johnston, University of California, San Francisco

Abstract number 22 Curative treatment and effective palliation with hemipelvectomy-A useful operation: modern concepts based on 20 years of experience-James C Wittig, Jacob Bickels, Felasfa Wodajo, Kristen L. Kellar-Graney, Yehuda Kollender, Isaac Meller, Martin M Malawer Division of Orthopedic Oncology, Washington Cancer Institute and National Unit of Orthopedic Oncology, Tel-Aviv Sourasky Medical Center Abstract number 16 (Young Investigator Award Winner) Bugged out? Infection and endoprosthetic replacements-Lee M Jeys, Raj Suneja, Vishnu Prasad, Robert Grimer, Simon R Carter, Roger Tillman Royal Orthopaedic Hospital Oncology Service, Royal Orthopaedic Hospital Summary and Panel Discussion: Where do we go from here? Thor Alvegard

12:15 p.m. Buffet Lunch, Poster Viewing

12:15 p.m Board of Directors Meeting

1:15 p.m. Jaap Verweij, CTOS President: Presentations of Two Young Investigator Awards (Lee M Jeys, Jonathan C Trent) 
1:30 p.m. SOFT TISSUE SARCOMAS: Chairmen: Jean-Yves Blay (France) \& Brian O'Sullivan (Canada)

1:30 p.m. Pancras Hogendoorn: Grading of soft tissue tumours

1:45 p.m. Peter Pisters: Preoperative chemoradiotherapy for locally advanced soft tissue sarcomas

2:00 p.m. Brian O'Sullivan: The advantage of new radiotherapy technologies in the management of soft tisssue sarcoma

2:15 p.m. Controversy session: polychemotherapy vs. monochemotherapy in advanced sarcomas:

2:15 p.m. pro: Bob Benjamin

2:30 p.m. contra: Jaap Verweij

2:45 p.m. Discussion

3:00 p.m. Abstract number 56 Margin width has prognostic significance for extremity and truncal sarcoma-Mark D.McKee, Dong Feng Liu, Deborah Driscoll, John J. Brooks, John F. Gibbs, William G. Kraybill Roswell Park Cancer Institute

3:15 p.m. Summary \& Panel Discussion: Where do we go from here? Jean Yves Blay

3:45 p.m. Coffee Break, Poster Viewing

4:00 p.m. BASIC SCIENCE/BIOLOGY: Jonathan Fletcher (USA) \& Junya Toguchida (Japan)

4:00 p.m.
4:20 p.m.
4:40 p.m.
5:00 p.m.

5:15 p.m.
Marc Ladanyi: Alveolar soft part sarcoma oncogene

Charlie Roberts: SNF5/INI1 haploinsufficiency predisposes to rhabdoid tumors in mice

Paul Meltzer: Clinical and biological applications of sarcoma gene expression profiling

Abstract number 106 Gene-expression profiles in chondrosarcomas and chordomas-Thomas F. DeLaney, Brian Seed, Ramnik Xavier, Andrew L. Rosenberg, G. Petur Nielsen, Francis J. Hornicek, Norbert J. Liebsch, John E. Munzenrider, Herman D. Suit, Massachusetts General Hospital Summary and Panel discussion: Jonathan Fletcher \& Junya Toguchida

6:00-6.30 p.m. Members' Business Meeting

7:15 p.m CTOS Reception and Dinner Banquet

Saturday, November 3, 2001

7:00 a.m. Registration, Breakfast for meeting attendees

8:00 a.m. NINA AXELRAD KEYNOTE LECTURE (Supported by the Nina Axelrod Sarcoma Fund):

Allan van Oosterom (Leuven, Belgium): Systemic treatment of advanced soft tissue sarcomas is not uniform any longer; Glivec as a paradigm.

9:00 a.m. IMATINIB MESYLATE (DEVELOPED AS STI-571): George Demitri (USA) \& Jaap Verweij (Netherlands)

9:00 p.m. Brian Rubin: Biology of GISTs

9:15 p.m. George Demitri: The USA experience

9:30 p.m. Jaap Verweij: The EORTC phase 2 study

9:45 p.m. Abstract number 131 (Young Investigator Award Winner) Inhibition of PDGF receptor tyrosine kinase in human soft-tissue sarcoma-Jonathan C. Trent, Jonathan Hannay, Lan Li, Shreyaskumar Patel, Robert S. Benjamin, Raphael E. Pollock, Di-Hua Yu The University of Texas, M. D. Anderson Cancer Center

10:00 p.m. Panel Discussion: Where do we go from here? George Demitri \& Jaap Verweij 
10:15 a.m. Coffee Break, Poster Viewing

10:30 a.m. Workshop: Genetics of Sarcoma: Jonathan Fletcher (USA) \& Lee Helman (USA)

10:30 a.m. Paola Dal Cin: Novel cytogenetic mechanisms in mesenchymal tumors

10:50 a.m. Marc Ladanyi: Specificity and clinical correlations for SYT-SSX

11:10 a.m. Abstract number 130 Expression profiling of osteosarcoma biopsies and metastases with CDNA microarrays-Luca Sangiorgi, Giuliana Alessandra Gobbi, Enrico Lucarelli, Francesca Scrimieri, Piero Picci, Paul Meltzer, Lab. Oncology Research, Rizzoli Orthopedic Institute, and Section of Molecular Genetics, Cancer Genetics Branch, National Human Genome Research Institute, National Institutes of Health

11:25 a.m. Summary and Panel Discussion: Where do we go from here? Jonathan Fletcher \& Lee Helman

11:45 a.m. Buffet Lunch, Poster Viewing

12:45 p.m. COOPERATIVE GROUP RESEARCH: Jaap Verweij (The Netherlands) \& Karen Antman (USA)

1:45 p.m. Mini Symposium: STAGING, RESPONSE ASSESSMENT \& FOLLOW-UP: Peter Hohenberger (Germany) \& Ian Judson (UK)

1:45 p.m. Jan Bloem: Staging and dynamic contrast enhanced MRI for response assessment

2:00 p.m. Peter Hohenberger: 31P magnetic resonance spectroscopy (31P-MRS) for non-invasive assessment of pathohistologic tumor response of soft tissue sarcoma

2:15 p.m. A. Kevin Raymond: Pathohistological response assessment following systemic chemotherapy for sarcomas

2:30 p.m. Summary and Panel Discussion: Where do we go from here? Ian Judson

2:45 p.m. CASE DISCUSSIONS: ANGIOSARCOMAS Paolo Casali (Italy)

Medical Oncology: Paolo Casali

Pathology: John Brooks

Surgery: Sylvie Bonvalot

Radiotherapy: Kirsten Sundby Hall

3:45 p.m. Adjournment 


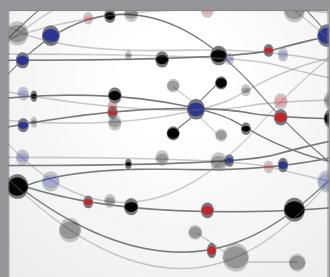

The Scientific World Journal
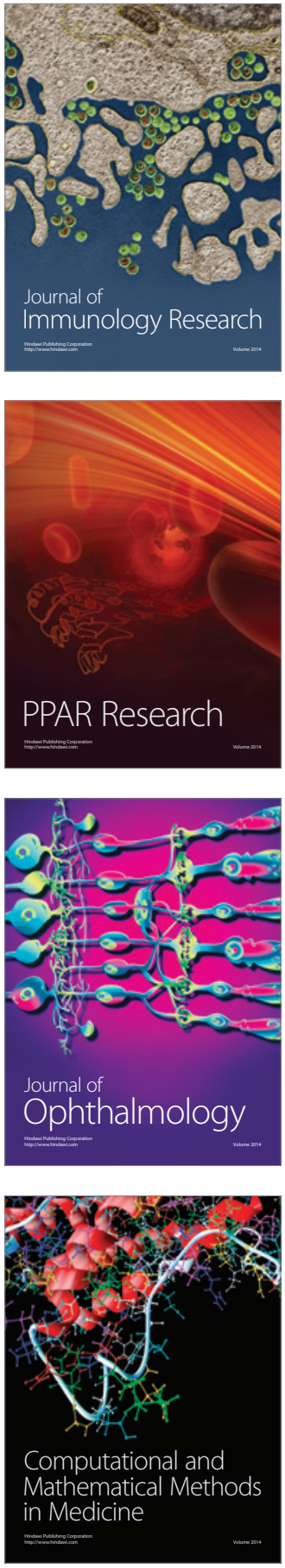

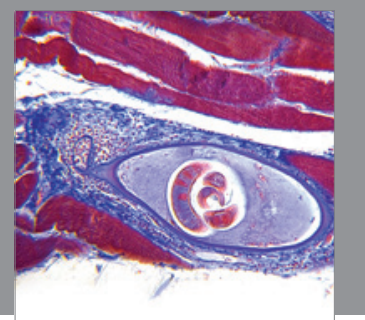

Gastroenterology

Research and Practice
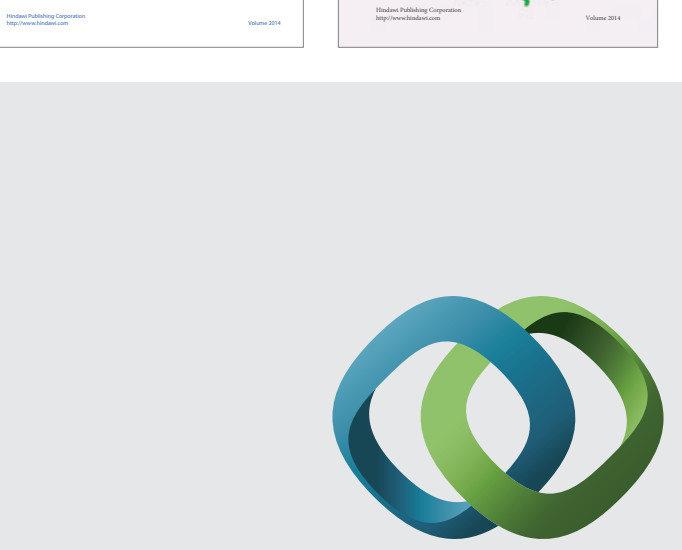

\section{Hindawi}

Submit your manuscripts at

http://www.hindawi.com
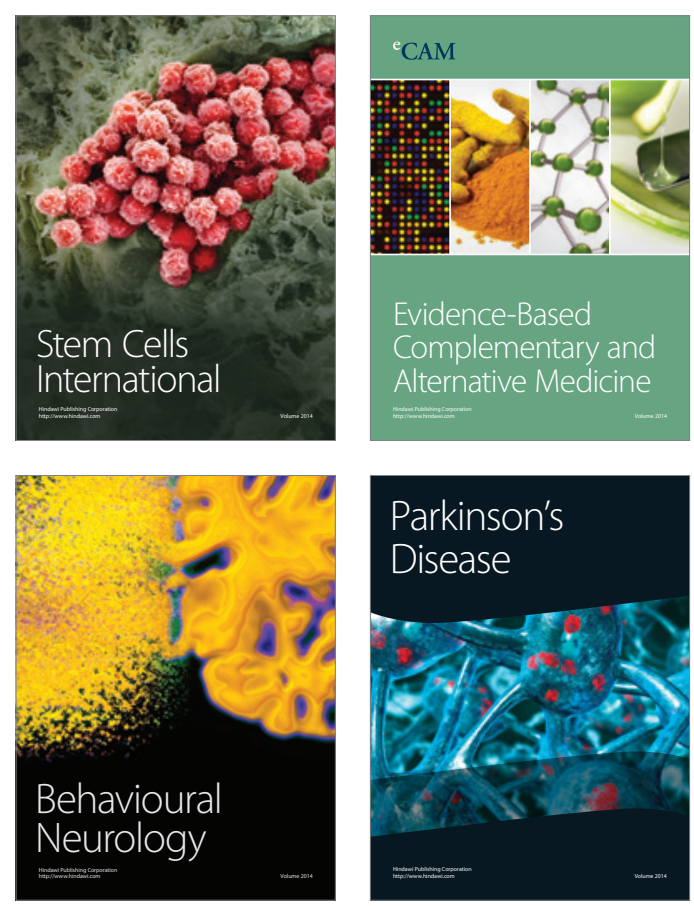

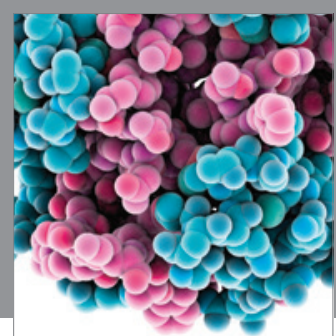

Journal of
Diabetes Research

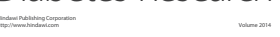

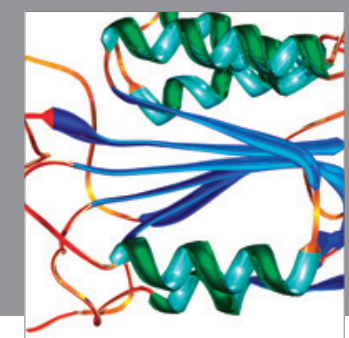

Disease Markers
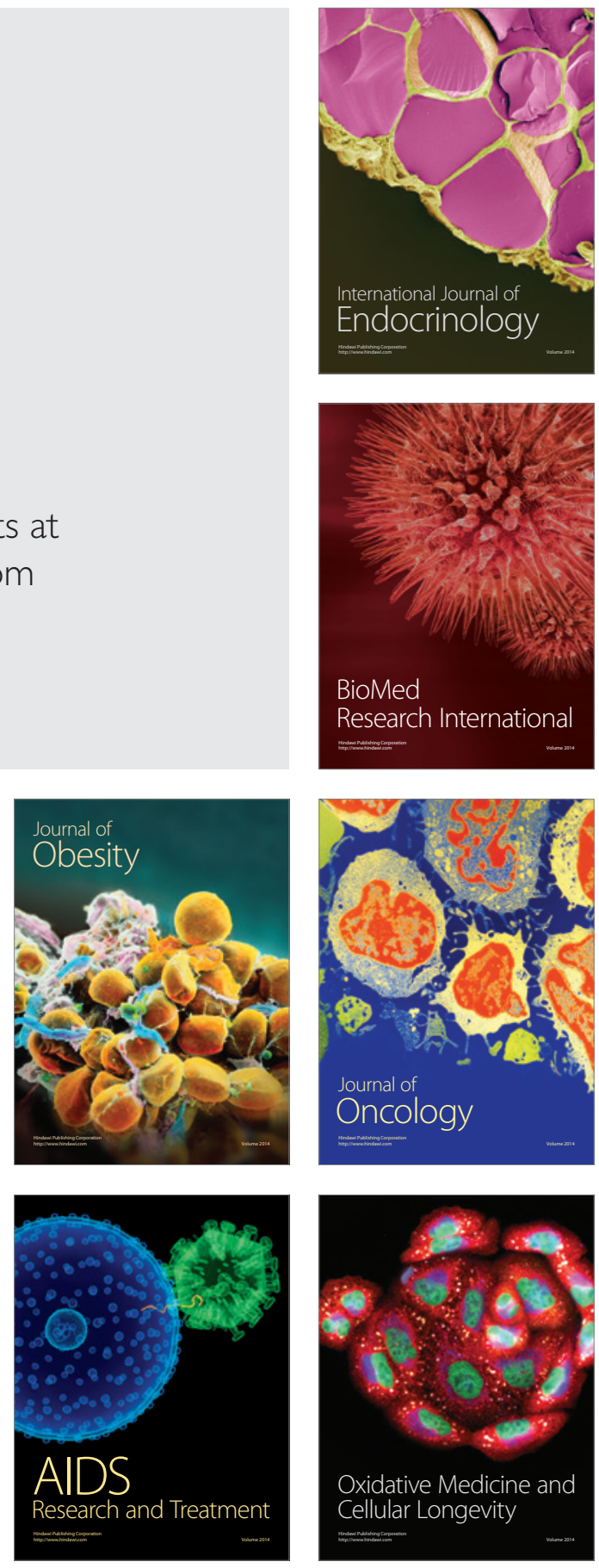